\title{
Codimension one immersions and the Kervaire invariant one problem
}

\author{
By PETER JOHN ECCLES \\ University of Manchester
}

(Received 30 March 1981)

\section{Introduction}

Let $i: M \hookrightarrow \longrightarrow \mathbb{R}^{n+1}$ be a self-transverse immersion of a compact closed smooth $n$-dimensional manifold in $(n+1)$-dimensional Euclidean space. A point of $\mathbb{R}^{n+1}$ is an $r$-fold intersection point of the immersion if it is the image under $i$ of (at least) $r$ distinct points of the manifold. The self-transversality of $i$ implies that the set of $r$-fold intersection points is the image of an immersion of a manifold of dimension $n+1-r$ (the empty set if $r>n+1)$. In particular, the set of $(n+1)$-fold intersection points is finite of order, say, $\theta(i)$. In this paper we are concerned with the set of values of $\theta(i)$ for (selftransverse) immersions of all (compact closed smooth) manifolds of given dimension $n$.

Placing $n+1$ copies of the $n$-sphere $S^{n}$ in general position in $\mathbb{R}^{n+1}$ provides an immersion $i_{0}: \cup S^{n} \uparrow \longrightarrow \mathbb{R}^{n+1}$ with $\theta\left(i_{0}\right)=2$. Thus given an immersion $i_{1}: M \uparrow \longrightarrow \mathbb{R}^{n+1}$ disjoint union with $i_{0}$ provides another immersion $i_{2}$ with $\theta\left(i_{2}\right)=\theta\left(i_{1}\right)+2$. On the other hand, given an immersion $i_{1}: M \uparrow \longrightarrow \mathbb{R}^{n+1}$ with $\theta\left(i_{1}\right) \geqslant 2$ handles may be attached to $M$ to eliminate two of the $(n+1)$-fold points giving an immersion $i_{2}$ with $\theta\left(i_{2}\right)=\theta\left(i_{1}\right)-2$ (the argument for $n=2$ is given in detail by T.F. Banchoff(2)). This leaves the problem of whether $\theta(i)$ can be odd.

In a previous paper (11) it was shown that if $M$ is orientable then $\theta(i)$ can be odd if and only if $n=0,1$ or 3 (see also (18)). The method of proof was to observe that the parity of $\theta$ is a bordism invariant and so to translate the problem into homotopy theory where it could be solved using standard techniques of algebraic topology. The same method can be applied in the general case, as explained in $\$ 2$, but so far has not led to a complete solution. However it does show that for certain values of $n$ the problem is equivalent to well known problems in homotopy theory. The main result of this paper is as follows.

THEOREM (a) When $n$ is even, $\theta(i)$ can be odd if and only if $n=0,2$ or 6 . (b) When $n$ is congruent to 1 modulo $4, \theta(i)$ can be odd if and only if there is a framed manifold of dimension $n+1$ with Kervaire invariant 1. (This requires $n+3$ to be a power of 2 and is true for $n=1,5,13(7), 29(19)$ and 61 (4).)

Part $(a)$ of the theorem is proved (in $\S 3$ ) by observing that, for even $n, \theta(i)$ can be odd if and only if $(n+1)$-dimensional real projective space $P^{n+1}$ is stably reducible. This is true if and only if there is an element in the $(n+1)$-stem $\pi_{n+1}^{S}$ of Hopf invariant one. Part $(b)$ is proved (in $\S 4$ ) by showing that, for these values of $n, \theta(i)$ can be odd if and only if there is an element of $\pi_{n+1}^{S}$ detected by a certain secondary cohomology 
operation, a problem known to correspond to the Kervaire invariant problem. Thus in each case the immersion problem considered here is equivalent to a famous problem in the homotopy groups of spheres. Although the theorem is proved in these homotopy theoretic terms some remarks on the construction of the immersions are included in $\S 5$.

For $n \equiv 3(\bmod 4)$ the only value of $n$ for which it is known that $\theta(i)$ can be odd is $n=3$ which arose in the orientable case; there is an immersion of the 3 -sphere in $\mathbb{R}^{4}$ with an odd number of 4 -fold points. Specific calculation in low dimensions and some more general considerations suggest that this is the only value of $n \equiv 3(\bmod 4)$ for which $\theta(i)$ can be odd. This is work in progress.

The results of this paper were announced in (10) which also contains a more leisurely introduction to the problem.

\section{Reducing the problem to homology theory}

In this section a slightly more general form of the problem posed in the introduction is reduced to an equivalent problem in the homology of certain infinite loop spaces. The key idea enabling this to be done is the geometric formulation of stable Hopf invariants given by U. Koschorke and B.J. Sanderson(17). A more detailed discussion of the ideas which follow in the case of oriented codimension one immersions may be found in ((11); $§ 2)$. That particular case is quite typical of the general case.

Suppose that $\xi$ and $\zeta$ are $k$-dimensional vector bundles. A bundle map $\xi \rightarrow \zeta$ will be referred to as a $\zeta$-structure on $\xi$. If $\zeta$ is the $k$-plane bundle associated with a universal $G$-bundle for a subgroup $G$ of $O(k)$, the group of $k$ by $k$ orthogonal matrices, then a $\zeta$-structure is usually described as a $G$-structure. In particular, if $\zeta$ is the bundle over a point, $G$ is the trivial group and a $\zeta$-structure is a trivialization or framing.

An immersion $i: M^{n} \longrightarrow \mathbb{R}^{n+k}$ of a compact closed smooth $n$-manifold will be called a $\zeta$-immersion if the normal bundle of the immersion has been given a $\zeta$-structure. If such an immersion is self-transverse and $n=k m$ then the set of $(m+1)$-fold intersection points is a finite set. If further $k$ is even and $\zeta$ is oriented then a sign may be attached to each intersection point by comparing the standard orientation of $\mathbb{R}^{n+k}$ with that provided by the orientations of the $m+1$ normal $k$-planes at the point. In this case let $\theta(i) \in \mathbb{Z}$ be the number of $(m+1)$-fold intersection points counted with signs. In other cases let $\theta(i) \in \mathbb{Z}_{2}(=\mathbb{Z} / 2 \mathbb{Z})$ be the number of $(m+1)$-fold intersection points modulo 2 . In either case $\theta$ gives a bordism invariant. The general problem is to determine the possible values for $\theta(i)$ given $n$ and $\zeta$.

To state an equivalent problem in homology theory some preliminaries are necessary. For any pointed space $X$ write $Q X$ for the direct $\operatorname{limit} \lim \Omega^{n} \Sigma^{n} X$ where $\Sigma$ is the $\vec{n}$

reduced suspension functor, $\Omega$ is the loop space functor and $\Omega^{n} \Sigma^{n} X \rightarrow \Omega^{n+1} \Sigma^{n+1} X$ is the standard inclusion. If $X$ is a connected space of the homotopy type of a $C W$ complex there is a natural stable homotopy equivalence(3), (23)

$$
Q X \equiv \bigvee_{r=1}^{\infty} D_{r} X
$$


Here $D_{r} X$ denotes the $r$-adic construction

$$
X_{\Sigma_{r}}^{\rtimes} W \Sigma_{r}=X^{(r)} \underset{\Sigma_{r}}{\times} W \Sigma_{r} /\{*\} \underset{\Sigma_{r}}{\times} W \Sigma_{r}
$$

where $\Sigma_{r}$ is the permutation group on $r$ objects, $W \Sigma_{r}$ is an acyclic space on which $\Sigma_{r}$ acts freely and $*$ is the base point of $X$. In particular $D_{1} X=X$. This equivalence implies that there is a natural isomorphism

$$
\tilde{H}_{i}(Q X) \cong \bigoplus_{r=1}^{\infty} \tilde{H}_{i}\left(D_{r} X\right),
$$

taking homology with any coefficients. Write

$$
p_{r}: \tilde{H}_{i}(Q X) \rightarrow \tilde{H}_{i}\left(D_{r} X\right)
$$

for the resulting projection map.

When $X=M \zeta$, the Thom complex of the $k$-plane bundle $\zeta, H_{k}(M \zeta)$ is generated by the Thom class $t$ of infinite order if $\zeta$ is orientable (in which case a choice of orientation determines the generator) and of order two if not. In either case $D_{r} X$ is $(k r-1)$ connected and the image of $t^{m+1} \in H_{k(m+1)}\left(X^{(m+1)}\right)$ in $H_{k(m+1)}\left(D_{m+1} X\right)$ under the map induced by the inclusion map (also denoted $t^{m+1}$ ) provides a generator for the group. If $\zeta$ is orientable and $k$ is even $t^{m+1} \in H_{k(m+1)}\left(D_{m+1} X\right)$ has infinite order and otherwise it is of order two (taking homology with integral coefficients) since its image in

$$
H_{k \neq m+1)}\left(D_{m+1} X ; \mathbb{Z}_{2}\right) \cong \mathbb{Z}_{2}
$$

is in the image of the Bockstein (which may be identified with the dual of the Steenrod square, $\left.S q_{*}^{1}\right)$.

Let $h: \pi_{k(m+1)}(Q M \zeta) \rightarrow H_{k^{\prime} m+1}(Q M \zeta)$ denote the Hurewicz homomorphism.

THEOREM 2-2. There exists a self-transverse $\zeta$-immersion $i: M^{k m} \longrightarrow \mathbb{R}^{k(m+1)}$ with $\theta(i)=\lambda$ if and only if there is an element $\alpha$ of $\pi_{k(m+1)}(Q M \zeta)$ such that $p_{m+1} h(\alpha)=\lambda t^{m+1}$.

The proof of this result is outlined at the end of the section.

When $t^{m+1}$ is of order two it is sufficient to work with homology with $\mathbb{Z}_{2}$-coefficients and in general this will determine the parity of $\theta(i)$. Then use can be made of the rich structure of the Hopf algebra $H_{*}\left(Q X ; \mathbb{Z}_{2}\right)$ over the Steenrod algebra $A_{2}$ which is explicitly known and is determined by the structure of the coalgebra $H_{*}(X)$ over $A_{2}$ (6), (8), (9). Its description uses the Kudo-Araki operations which are homomorphisms

$$
Q^{i}: H_{n}\left(Q X ; \mathbb{Z}_{2}\right) \rightarrow H_{n+i}\left(Q X ; \mathbb{Z}_{2}\right),
$$

which are trivial for $i<n$ and equal to the Pontrjagin square for $i=n$. Now for $X$ connected, if $\left\{x_{\alpha}\right\}$ is a homogeneous basis for $\tilde{H}_{*}\left(X ; \mathbb{Z}_{2}\right) \subset H_{*}\left(Q X ; \mathbb{Z}_{2}\right)$, a basis for $H_{*}\left(Q X ; \mathbb{Z}_{2}\right)$ is provided by the set of monomials in elements of the form

$$
Q^{I} x_{\alpha}=Q^{i_{1}} Q^{i_{2}} \ldots Q^{i_{r}} x_{\alpha},
$$

where $I=\left(i_{1}, i_{2}, \ldots, i_{\tau}\right)$ is an admissible sequence (i.e. $i_{j} \leqslant 2 i_{j+1}$ for $1 \leqslant j<r$ ) of excess (i.e. $i_{1}-i_{2}-\ldots-i_{r}$ ) greater than the dimension of $x_{\alpha}$. This basis will be referred to as a Dyer-Lashofbasis for $H_{*}\left(Q X ; \mathbb{Z}_{2}\right)$. The Hopf algebra structure is given by the coproduct 
Cartan formula, the action of the Kudo-Araki operations is given by the Adem relations and the product Cartan formula, and the action of the Steenrod algebra is determined by the Nishida relations. These various formulae are conveniently listed as ((8), Theorem I.1·1). Furthermore if we define a height function $h$ on the Dyer-Lashof basis by $h\left(x_{\alpha}\right)=1, h\left(Q^{i \xi}\right)=2 h(\xi)$ and $h(\xi \eta)=h(\xi)+h(\eta)$ then under the isomorphism (2.1) the basis elements of height $r$ provide a basis for $\tilde{H}_{*}\left(D_{r} X\right)((21)$, Proposition 2.4). Thus Theorem $2 \cdot 2$ can be reformulated as follows.

Theorem 2.3. There exists a self-transverse $\zeta$-immersion $i: M^{k m} \uparrow \longrightarrow \mathbb{R}^{k(m+1)}$ with $\theta(i)$ odd if and only if there is an element of $\pi_{k(m+1)}(Q M \zeta)$ whose Hurewicz image in $H_{k(m+1)}\left(Q M \zeta ; \mathbb{Z}_{2}\right)$ involves $t^{m+1}$.

Here a homology class involves $t^{m+1}$ when $t^{m+1}$ has a non-zero coefficient when the class is written in terms of a Dyer-Lashof basis.

This theorem is equivalent to Theorem $2 \cdot 2$ when $\theta(i)$ is $\mathbb{Z}_{2}$-valued, i.e. when $k$ is odd or $\zeta$ is non-orientable. In this paper only the case $k=1$ is considered and so this is sufficient. However interesting results can be obtained in other cases where Theorem $2 \cdot 2$ is needed, for example when $k=2$ and $\zeta$ is the universal SO(2)-bundle.

Theorem 2.2 is proved by observing, after R. Wells(25), that the bordism group of $\zeta$-immersions of compact closed smooth $n$-manifolds in $\mathbb{R}^{n+k}$ is isomorphic to the homotopy group $\pi_{n+k}(Q M \zeta)$ ((11), Proposition 2.1). Thus for $n=k m$ the order of the $(m+1)$-fold intersection set of such a self-transverse immersion determines a homomorphism

$$
\theta: \pi_{k(m+1)}(Q M \zeta) \rightarrow \mathbb{Z} \text { or } \mathbb{Z}_{2} .
$$

Now, following U. Koschorke and B. J. Sanderson(17), this map may be identified with a stable Hopf invariant

$$
\left(h_{M \zeta}^{m+1}\right)_{*}: \pi_{k(m+1)}(Q M \zeta) \rightarrow \pi_{k l m+1)}\left(Q D_{m+1} M \zeta\right)
$$

as defined in ((3), §5) or (23). This is a special case of ((11), Proposition 2.2). The result now follows once we observe that by the definition of $h_{M \zeta}^{m+1}$ the following diagram commutes.

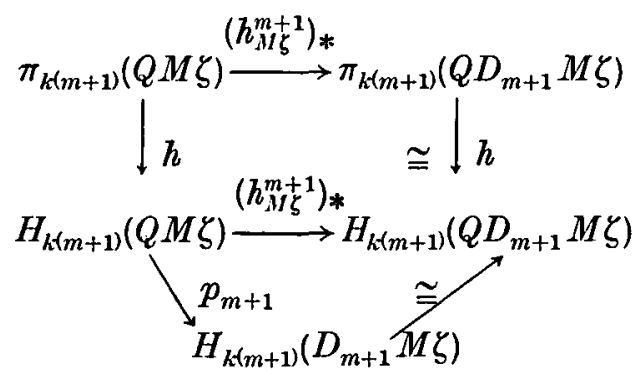

The right-hand maps are isomorphisms since $D_{m+1} M \zeta$ is $((m+1) k-1)$-connected.

3. Primitive classes in $H_{*}\left(Q P^{\infty} ; \mathbb{Z}_{2}\right)$ : the main theorem for even $n$

The main theorem is proved by applying Theorem $2 \cdot 3$. When $\zeta$ is the universal (real) line bundle, $M \zeta$ is infinite-dimensional real projective space $P^{\infty}$. Throughout the remainder of this paper $H_{*}$ denotes homology with $\mathbb{Z}_{2}$-coefficients and $a_{i}$ denotes the 
non-zero element in $\tilde{H}_{i}\left(P^{\infty}\right) \cong \mathbb{Z}_{2}$ for $i \geqslant 1$. In particular $a_{1}$ is the Thom class of the bundle $\zeta$.

Theorem $2 \cdot 3$ is applied by observing that a spherical class $\alpha$ in $H_{n+1}\left(Q P^{\infty}\right)$, i.e. an element in the image of the Hurewicz homomorphism, is necessarily primitive with respect to the cup coproduct $\psi$, i.e.

$$
\psi(\alpha)=\alpha \otimes 1+1 \otimes \alpha .
$$

The structure of the primitives in $H_{*}\left(Q P^{\infty}\right)$ is rather complicated and a description is not attempted here. All that is required is the following simple result about primitives which involve $a_{1}^{n+1}$.

Lemma 3-1. Suppose that $n+1=p q$ where $p$ is a power of two and $q$ is odd. Then $a$ primitive class in $H_{n+1}\left(Q P^{\infty}\right)$ involves $a_{1}^{n+1}$ if and only if it involves $a_{q}^{p}$.

This is proved at the end of the section. It allows us to reformulate Theorem $2 \cdot 3$ in terms of classes of lower height.

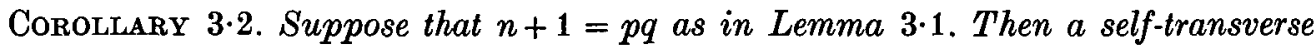
immersion $i: M^{n} \uparrow \longrightarrow \mathbb{R}^{n+1}$ has $\theta(i)$ odd if and only if the corresponding element of $\pi_{n+1}\left(Q P^{\infty}\right)$ has Hurewicz image involving $a_{q}^{p}$.

When $n$ is even this shows that the intersection point question is equivalent to the stable reducibility of $(n+1)$-dimensional real projective space $P^{n+1}$. For if $\alpha \in \pi_{n+1}\left(Q P^{n+1}\right)$ is such a stable reduction then its Hurewicz image in

$$
H_{n+1}\left(Q P^{n+1}\right) \subset H_{n+1}\left(Q P^{\infty}\right)
$$

involves $a_{n+1}$. Conversely an element of $\pi_{n+1}\left(Q P^{\infty}\right)$ whose Hurewicz image involves $a_{n+1}$ may be pulled back to a stable reduction in $\pi_{n+1}\left(Q P^{n+1}\right)$. However it is well-known that $P^{n+1}$ is stably reducible if and only if $n=0,2$ or 6 (1). This completes the proof of the main theorem for even $n$.

To prove Lemma $3 \cdot 1$, first observe that it is immediate for $p=1$ (all that has been used in the above argument) from the observation that a primitive class in $H_{q}\left(Q P^{\infty}\right)$ involves $a_{1}^{i} a_{q-i}$ if and only if it involves $a_{1}^{i-1} a_{q-i+1}$ (for $1 \leqslant i \leqslant q-1$ ). This in turn follows from the fact that these are the only two elements of $H_{q}\left(Q P^{\infty}\right)$ involving $a_{1}^{i} \otimes a_{q-i}$ in their coproducts (this uses $q$ odd when $i=q-1$ ).

The general result (which is needed later) follows from this using the fact that a decomposable primitive is a square ((20), Proposition 4.21). For let $A$ denote the polynomial algebra $\mathbb{Z}_{2}\left[a_{i} \mid i \geqslant 1\right]$. This is a subalgebra of $H_{*}\left(Q P^{\infty}\right)$ : write $B$ for its orthogonal complement with respect to the Dyer-Lashof basis. Then $H_{*}\left(Q P^{\infty}\right)=A \oplus B$ and the coproduct gives maps

$$
\begin{aligned}
& \psi: A \rightarrow A \otimes A, \\
& \psi: B \rightarrow A \otimes B+B \otimes A+B \otimes B,
\end{aligned}
$$

by the Cartan formulae ((8), Theorem I·1·1 (6)). Thus, for $\alpha=\alpha_{1}+\alpha_{2} \in H_{*}\left(Q P^{\infty}\right)$ where $\alpha_{1} \in A, \alpha_{2} \in B, \alpha$ is primitive if and only if $\alpha_{1}$ and $\alpha_{2}$ are primitive. This means that we may suppose, without loss of generality, that the primitive class referred to in the lemma is an element of $\mathbb{Z}_{2}\left[a_{i} \mid i \geqslant 1\right]$.

However, if $n+1$ is even (say $n+1=2 m$ ), $a_{n+1}$ cannot be involved in a primitive 
class for it is the only basis element whose coproduct involves $a_{m} \otimes a_{m}$. Thus in this case any $(n+1)$-dimensional primitive of $\mathbb{Z}_{2}\left[a_{i} \mid i \geqslant 1\right]$ is decomposable and so, as remarked above, is the square of a primitive.

Now suppose that $p=2^{l}, l \geqslant 0$. The result follows from the above argument by induction on $l$.

\section{The real reflection map: the main theorem for $n \equiv 1(\bmod 4)$}

The relationship between the problem under consideration and the Kervaire invariant one problem is provided by the real reflection map $\lambda: P^{\infty} \rightarrow Q S^{0}$. This is defined, up to homotopy, as the direct limit of maps $\lambda_{n}: P^{n} \rightarrow \Omega^{n+1} S^{n+1}$ given by $\lambda_{n}(x)=\lambda_{n}^{\prime}(x) * 1$. Here $\lambda_{n}^{\prime}(x): S^{n+1} \rightarrow S^{n+1}$ is the one point compactification of the map $\mathbb{R}^{n+1} \rightarrow \mathbb{R}^{n+1}$ given by reflection in the hyperplane orthogonal to $x \in P^{n}$, denotes the track sum and $1: S^{n+1} \rightarrow S^{n+1}$ is the identity map. Using the canonical infinite loop structure of $Q S^{0}, \lambda$ may be extended to a map $\lambda: Q P^{\infty} \rightarrow Q S^{0}$. The induced map in homotopy $\lambda_{*}: \pi_{n+1}\left(Q P^{\infty}\right) \rightarrow \pi_{n+1}\left(Q S^{0}\right)$ is onto the 2-component for $n \geqslant 0$ by the Kahn-Priddy Theorem (15).

The main theorem for $n \equiv 1(\bmod 4)$ now follows from Corollary 3.2 and the following result.

Proposition 4·1. If $n \equiv 1(\bmod 4)$ and $n+1=2 m$, an element $\alpha \in \pi_{n+1}(Q P \infty)$ has Hurewicz image involving $a_{m}^{2}$ if and only if $m+1$ is a power of 2 , say $m+1=2^{j}$, and $\lambda_{*}(\alpha) \in \pi_{n+1}\left(Q S^{0}\right) \cong \pi_{n+1}^{S}$ is detected by the secondary cohomology operation $\phi_{j, j}$.

For it is well-known (7) that elements detected by $\phi_{j, j}$ correspond under the Pontrjagin(-Thom) construction to framed manifolds of dimension $n+1$ with Kervaire invariant one.

Here $\phi_{j, j}$ is the secondary cohomology operation based on the Adem relation

in the $\bmod 2$ Steenrod algebra.

$$
S q\left(2^{j}\right) S q\left(2^{j}\right)=\sum_{k=0}^{j-1} S q\left(2^{j+1}-2^{k}\right) S q\left(2^{k}\right)
$$

Of course the result of the previous section for $n$ even can be formulated in the same way. In that case $\alpha \in \pi_{n+1}\left(Q P^{\infty}\right)$ represents an immersion $i$ with $\theta(i)$ odd if and only if $\lambda_{*}(\alpha) \in \pi_{n+1}^{S}$ is detected by $S q^{n+2}$, i.e. has (Steenrod-) Hopf invariant one.

To prove the proposition we again reformulate the method of detecting $\alpha$. It should cause no confusion if $a_{m}$ is also used to denote the generator of $H^{m}\left(P^{\infty}\right)$. The observation that a certain James-Hopf invariant is equivalent to the Steenrod-Hopf invariant gives the following result.

LEMMA 4.2. $\alpha \in \pi_{2 m}\left(Q P^{\infty}\right)$ has Hurewicz image involving $a_{m}^{2}$ if and only if its stable adjoint $\alpha \in \pi_{2 m}^{S}\left(P^{\infty}\right)$ is detected by the functional cohomology operation $S q_{\alpha}^{m+1}$ on $a_{m} \in H^{m}\left(P^{\infty}\right)$.

This may be used first to obtain the restriction on $m$.

Lemma 4.3. An element $\alpha \in \pi_{2 m}^{S}\left(P^{\infty}\right)$ may be detected by $S q_{\alpha}^{m+1}$ on $a_{m}$ only if $m+1$ is a power of 2 .

Finally the first Peterson-Stein formula may be used to prove the following. 
LEMмA 4.4. If $m=2^{j}-1$, then $\alpha \in \pi_{2 m}^{S}\left(P^{\infty}\right)$ is detected by $S q_{\alpha}^{m+1}$ on $a_{m}$ if and only if $\lambda_{*}(\alpha) \in \pi_{2 m}^{S}$ is detected by $\phi_{j, j}$.

These results imply Proposition $4 \cdot 1$. It remains to prove them.

Write $P_{m}^{\infty}$ for the truncated projective space $P^{\infty} / P^{m-1}$. Since the quotient map $P^{\infty} \rightarrow P_{m}^{\infty}$ induces an isomorphism of homology and cohomology groups of dimension $m$ and above, Lemma $4 \cdot 2$ is equivalent to the same statement with $P^{\infty}$ replaced by $P_{m}^{\infty}$ where $a_{m}$ denotes the corresponding homology and cohomology classes in $P_{m}^{\infty}$.

Now observe that for dimensional reasons $\Omega \Sigma P_{m}^{\infty} \rightarrow Q P_{m}^{\infty}$ is a $2 m$-equivalence. Lemma $4 \cdot 2$ is therefore equivalent to the statement that $\alpha \in \pi_{2 m}\left(\Omega \Sigma P_{m}^{\infty}\right)$ has Hurewicz image (involving) $a_{m}^{2}$ if and only if its adjoint $\hat{\alpha} \in \pi_{2 m+1}\left(\sum P_{m}^{\infty}\right)$ is detected by the functional cohomology operation

$$
S q_{\alpha}^{m+1}: H^{m+1}\left(\sum P_{m}^{\infty}\right) \rightarrow H^{2 m+1}\left(S^{2 m+1}\right)
$$

applied to the suspended class $\sigma a_{m}$, i.e. $\hat{\alpha}$ has Steenrod-Hopf invariant one.

In the following commutative diagram the vertical maps $h$ are Hurewicz maps and the horizontal maps are induced by the James map $\Omega \Sigma X \rightarrow \Omega \Sigma(X \wedge X)$ (13) with $X=P_{m}^{\infty}$ so that $J$ is the James-Hopf invariant.

$$
\begin{gathered}
\pi_{2 m}\left(\Omega \Sigma P_{m}^{\infty}\right) \stackrel{J}{\rightarrow} \pi_{2 m}\left(\Omega \Sigma P_{m}^{\infty} \wedge P_{m}^{\infty}\right) \\
\left.\downarrow_{H_{2 m}\left(\Omega \Sigma P_{m}^{\infty}\right) \rightarrow H_{2 m}\left(\Omega \Sigma P_{m}^{\infty} \wedge P_{m}^{\infty}\right)} h\right|^{\infty} h
\end{gathered}
$$

Since the right-hand map is an isomorphism and the bottom one maps $a_{m}^{2}$ to the generator and $a_{2 m}$ to $0, h(\alpha)$ involves $a_{m}^{2}$ if and only if the James-Hopf invariant $J(\alpha)$ in non-zero.

Lemma 4.2 now follows from the observation that this James-Hopf invariant is equivalent to the Steenrod-Hopf invariant ((5), Corollary 5·15).

The other two results must be well-known but there does not appear to be a suitable reference.

It is easier to prove a more general result than Lemma 4.3.

Lemma 4.5. Suppose that $n \equiv 2^{p}\left(\bmod 2^{p+1}\right)$ and $\alpha \in \pi_{n+m-1}^{S}\left(P^{\infty}\right)$. Write $C_{\alpha}$ for the mapping cone of $\alpha$. Then $S q^{n} a_{m}=0$ in $H^{*}\left(C_{\alpha}\right)$ when (i) $m \equiv 2^{p}-1\left(\bmod 2^{p+1}\right), m>2^{p}-1$, or (ii) $m \leqslant n-2^{p+1}$.

Since functional cohomology operations may be identified with cohomology operations in the mapping cone, Lemma 4.3 follows from part (i) of this result with $n=m+1$. Part (ii) is used below in the proof of Lemma $4 \cdot 4$.

Lemma 4.5 is an exercise in the Adem relations. Part (i) may be proved by induction on $p$. For $p=0$,

$$
\begin{aligned}
S q^{n} a_{m}=S q^{1} S q^{n-1} a_{m} \quad(n \text { odd })=\epsilon S q^{1} a_{m+n-1} & (\epsilon=0 \text { or } 1) \\
=\epsilon S q^{1} S q^{1} a_{m+n-2} & (m+n-1 \text { even })=0 \quad \text { (Adem) }
\end{aligned}
$$

For $p>0$, the inductive step is provided by first proving that $S q\left(2^{p}\right) a_{m}=0$ by the inductive hypothesis using the Adem relation arising from $S q\left(2^{p}\right) S q\left(2^{p}\right)$ and 
$a_{m}=S q\left(2^{p}\right) a_{m-2^{p}}$. Now if $n \equiv 2^{p}\left(\bmod 2^{p+1}\right)$ and $n>2^{p}$, the Adem relations allow us to write

$$
S q(n)=S q\left(2^{p}\right) S q\left(n-2^{p}\right)+\sum_{q=0}^{p-1} S q\left(n-2^{q}\right) S q\left(2^{q}\right)
$$

and so the general case follows from the special case again using the inductive hypothesis. The proof of part (ii) is by a similar induction on $p$.

Finally to prove Lemma 4.4 the following well-known result concerning the stable $\operatorname{map} \lambda: P^{\infty} \rightarrow S^{0}$ is required.

Proposition 4.6. $S q_{\lambda}^{i+1}\left(g_{0}\right)=a_{i}$ for all $i \geqslant 1$ where $S q_{\lambda}^{i+1}: \tilde{H}^{0}\left(S^{0}\right) \rightarrow \tilde{H}^{i}\left(P^{\infty}\right)$ is the functional cohomology operation and $g_{0} \in \tilde{H}^{0}\left(S^{0}\right)$ is the generator.

This is another exercise using the Adem relations. The result holds for $i=1$ since the restriction of $\lambda$ to $P^{1} \cong S^{1}$ is the Hopf map. The result can now first be proved for $i=2^{p}$ by induction on $p$. This provides the starting point of a proof of the general result by induction on the number of 1 's in the dyadic expansion of $2^{p}-i$ where $2^{p-1}<i \leqslant 2^{p}$. For if $i \equiv 2^{q}\left(\bmod 2^{q+1}\right)(0 \leqslant q<p-1), 2^{p}-\left(i+2^{q}\right)$ has one less 1 in its dyadic expansion than $2^{p}-i$. The inductive step is provided by

$$
S q\left(2^{q}\right) S q(i+1)_{\lambda}\left(g_{0}\right)=S q\left(i+2^{q}+1\right)_{\lambda}\left(g_{0}\right),
$$

the other terms in the Adem relation being zero for dimensional reasons.

Returning to Lemma 4.4, the first Peterson-Stein formula ((22); theorem 6.1) implies that $\lambda_{*}(\alpha) \in \pi_{2}^{S} \boldsymbol{S}_{j+1-2}$ is detected by the secondary operation $\phi_{j, j}$ if and only if $\sum_{k=1}^{j} S q\left(2^{j+1}-2^{k}\right) a_{2^{k}-1} \neq 0$ in $H^{*}\left(C_{\alpha}\right)$. The lemma now follows from the observation that $S q\left(2^{j+1}-2^{k}\right) a_{2_{-1}}=0$ for $1 \leqslant k<j$ by part (ii) of Lemma $4 \cdot 5$.

This completes the proof of Proposition 4.1 and so of the main theorem.

\section{The construction of immersions}

We have shown that, for even $n$, there is an immersion $i: M q \longrightarrow \mathbb{R}^{n+1}$ of an $n$-dimensional manifold with $\theta(i)$ odd if and only if $P^{n+1}$ is stably reducible. For even $n$ this is also a necessary and sufficient condition for $P^{n}$ to jmmerse in $\mathbb{R}^{n+1}((12)$, theorem 7.2). In fact any such immersion of $P^{n}$ will have $\theta(i)$ odd. For $n=2$ this is a consequence of T. F. Banchoff's theorem (2) that $\theta(i)$ has the same parity as the Euler characteristic of the immersed surface. Boy's surface provides such an immersion (10). In fact the same proof holds for $n=6$ since B. Hill-Tout has generalized Banchoff's theorem to all even dimensional manifolds. Alternatively, consider the commutative diagram

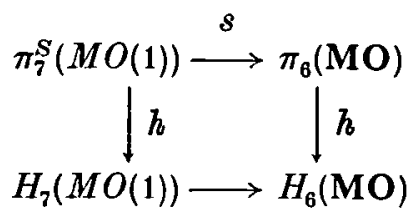

where $\mathrm{MO}$ is the $M O$-spectrum (so that $\pi_{*}(\mathrm{MO})$ is the unoriented bordism ring), the horizontal maps are stabilization maps (so that the upper map is induced by 
forgetting the immersion of an immersed manifold) and the vertical maps are Hurewicz homomorphisms. Suppose $x \in \pi_{7}^{S}(M O(1))$ is represented by an immersion $i: P^{6} \rightarrow \mathbb{R}^{7}$. It is well-known that $P^{6}$ represents a non-zero element of $\pi_{6}(\mathrm{MO})$ and that the Hurewicz map for MO is a monomorphism (24). Thus $h(x) \neq 0$ so that $h(x)=a_{7}$. It follows from Corollary $3 \cdot 2$ and the remarks following it that $\theta(i)$ is odd.

For $n \equiv 1(\bmod 4)$, it is possible to describe immersions with $\theta(i)=1$ for $n=1,5$ and 13. Thus for $m=1,3$ or 7 choose an immersion $i_{1}$ of $S^{m}$ in $\mathbb{R}^{2 m}$ with a trivialized normal bundle and an odd number of double points (i.e. representing an element $\alpha$ of $\pi_{2 m}\left(Q S^{m}\right)$ $\cong \pi_{m}^{S}$ of Hopf invariant 1) and any immersion $i_{2}$ of $P^{m-1}$ in $\mathbb{R}^{m}$ (i.e. a representative of $\beta \in \pi_{m}^{S}\left(P^{\infty}\right)$ such that $\left.h(\beta)=a_{m} \in H_{m}\left(P^{\infty}\right)\right)$. Then $\beta \circ \alpha \in \pi_{2 m}^{S}\left(P^{\infty}\right)$ is represented by an immersion $i: S^{m} \times P^{m-1} \longrightarrow \longrightarrow \mathbb{R}^{2 m}$ constructed via a tubular neighbourhood of $i_{1}$ by immersing $P^{m-1}$ by $i_{2}$ in each normal fibre of $i_{1}$ identified with $\mathbb{R}^{m}$ by the trivialization. Furthermore $\beta \circ \alpha$ is detected by $S q^{m+1}$ on $a_{m}$ and so $\theta(i)=1$ by Corollary 3.2 and Lemma 4.2. For $n=29$ and $n=61$ it does not appear to be known which manifold to take.

More generally it is possible to interpret the homotopy theory geometrically and so to give a direct geometrical correspondence between immersions $i: M \uparrow \longrightarrow \mathbb{R}^{n+1}$ and $(n+1)$-dimensional manifolds with a trivialized stable normal bundle so that, for $n \equiv 1(\bmod 4), \theta(i)$ corresponds to the Kervaire invariant.

In one direction we use the geometrical interpretation of the real reflection map $\lambda_{*}: \pi_{n+1}^{S}\left(P^{\infty}\right) \rightarrow \pi_{n+1}^{S}$ which has been provided by B. Gray and U. Koschorke ((16), $\S 2)$ as follows. Suppose that $i: M \uparrow \longrightarrow \mathbb{R}^{n+1}$ is an immersion of an $n$-dimensional manifold. Let $v$ be the normal bundle of the immersion

$$
i: M \hookrightarrow \mathbb{R}^{n+1} \cong \mathbb{R}^{n+1} \times\{0\} \subset \mathbb{R}^{n+2}
$$

An immersion of the total space $N$ of the circle bundle of $\nu$ may be obtained by placing a figure eight in each normal plane with vertical axis parallel to the last axis of $\mathbb{R}^{n+2}$. Koschorke writes this $8_{i}: N q \longrightarrow \mathbb{R}^{n+2}$. Clearly $N$ is orientable and so after a choice of orientation represents an element of $\pi_{n+2}^{S}\left(Q S^{1}\right) \cong \pi_{n+1}^{S}$. Gray and Koschorke have proved that if $i$ represents $\alpha \in \pi_{n+1}^{S}\left(P^{\infty}\right)$ then $8_{i}$ represents $\lambda_{*}(\alpha) \in \pi_{n+1}^{S}$. Thus the remarks in $\S 4$ show that $\theta(i)(\bmod 2)$ is the Kervaire invariant of $N$ with the trivialization of its stable normal bundle provided by $\boldsymbol{8}_{i}$.

Conversely the right inverse (at the prime 2) of $\lambda_{*}$ may also be interpreted geometrically. This is provided (14) by the stable Hopf invariant

$$
\left(h_{S 1}^{2}\right)_{*}: \pi_{n+2}\left(Q S^{1}\right) \rightarrow \pi_{n+2}\left(Q D_{2} S^{1}\right) \cong \pi_{n+1}\left(Q P^{\infty}\right)
$$

(3) observing that $D_{2} S^{1}$ is homeomorphic to $\Sigma P^{\infty}$. The identification of this map with the double point invariant of a codimension one immersion is another application of Koschorke and Sanderson's work. Given an oriented self-transverse immersion

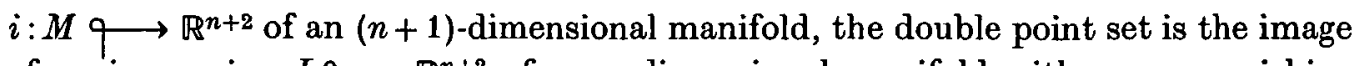
of an immersion $L \uparrow \longrightarrow \mathbb{R}^{n+2}$ of an $n$-dimensional manifold with a non-vanishing normal vector field provided by taking the average of the two normal vectors provided 
at each point of $L$ by the orientation of $M((11), \S 2)$. By Hirsch theory ((12), theorem 6.4) this is regularly homotopic to a (self-transverse) immersion

$$
\psi_{2}(i): L q \longrightarrow \mathbb{R}^{n+1} \cong \mathbb{R}^{n+1} \times\{0\} \subset \mathbb{R}^{n+2} .
$$

By ((11), proposition 2.2) if $i$ represents $\beta \in \pi_{n+1}\left(Q S^{1}\right)$ then $\psi_{2}(i)$ represents

$$
h_{*}^{2}(\beta) \in \pi_{n+1}\left(Q P^{\infty}\right),
$$

i.e. an element mapped by $\lambda_{*}$ to $\beta$ (modulo odd torsion). Thus $\theta\left(\psi_{2}(i)\right)$ is the Kervaire invariant of $M$ with the trivialization of its stable normal bundle provided by $i$.

\section{REFERENCES}

(1) Adams, J. F. On the non-existence of elements of Hopf invariant one. Ann. of Math . (2) 72 (1960), 20-104.

(2) Banchoff, T. F. Triple points and surgery of immersed surfaces. Proc. Amer. Math. Soc. 46 (1974), 407-413.

(3) Barrati, M. G. and Eccles, P.J. $\Gamma^{+}$-structures III: The stable structure of $\Omega^{\infty} \Sigma^{\infty} A$. Topology 13 (1974), 199-207.

(4) Barratt, M. G. and Mahowald, M. E. Unpublished.

(5) Boardman, J. M. and Steer, B. On Hopf invariants. Comment. Math. Helv. 42 (1967), 180-221.

(6) Browder, W. Homology operations and loop spaces. Illinois. J. Math. 4 (1960), 347357.

(7) BrowDER, W. The Kervaire invariant of framed manifolds and its generalization. Ann. of Math. (2) 90 (1969), 157-186.

(8) Cohen, F. R., LAdA, T. J. and MAY, J. P. The homology of iterated loop spaces. Lecture Notes in Math. no. 533 (Springer-Verlag, Berlin, Heidelberg, New York, 1976).

(9) Dyer, E. and Lashof, R. K. Homology of iterated loop spaces. Amer. J. Math. 84 (1962), $35-88$.

(10) Eccles, P. J. Multiple points of codimension one immersions. Topology Symposium Siegen 1979, pp. 23-38. Lecture Notes in Math. no. 788 (Springer-Verlag, Berlin, Heidelberg, New York, 1980).

(11) Eccles, P. J. Multiple points of codimension one immersions of oriented manifolds. Math. Proc. Cambridge Philos. Soc. 87 (1980), 213-220.

(12) Hirsch, M. W. Immersions of manifolds. Trans. Amer. Math. Soc. 93 (1959), 242-276.

(13) J AMES, I. M. On the suspension triad. Ann. of Math. (2) 63 (1956), 191-247.

(14) KAHN, D. S. Homology of the Barratt-Eccles decomposition maps. Conference on Homotopy Theory Evanston 1974, pp. 65-82. Notas de Matemática y Simposia no. 1 (Soc. Mat. Mexicana, Mexico, 1975).

(15) Kahn, D. S. and Priddy, S. B. The transfer and stable homotopy theory. Math. Proc. Cambridge Philos. Soc. 83 (1978), 103-111.

(16) Koschorke, U. Multiple points of immersions, and the Kahn-Priddy theorem. Math. Z. 169 (1979), 223-236.

(17) Koschorke, U. and Sanderson, B.J. Self-intersections and higher Hopf invariants. Topology 17 (1978), 283-290.

(18) LANNES, J. Immersions et homomorphisme d'Hurewicz (to appear).

(19) Mahowald, M. E. and Tangora, M. C. Some differentials in the Adams spectral sequence. Topology 6 (1967), 349-369.

(20) Mrtnor, J. and Moore, J.C. On the structure of Hopf algebras. Ann. of Math. (2) 81 (1965), 211-264.

(21) Nishid A, G. The nilpotency of elements of the stable homotopy groups of spheres. J. Math. Soc. Japan 25 (1973), 707-732.

(22) Peterson, F. P. and Stein, N. Secondary cohomology operations: two formulas. Amer. J. Math. 81 (1959), 281-305. 
(23) SNaITh, V. P. A stable decomposition of $\Omega^{n} S^{n} X$. J. London Math. Soc. (2) 7 (1974), 577-583.

(24) Tном, R. Quelques propriétés globales des variétés différentiables. Comment. Math. Helv. 28 (1954), 17-86.

(25) Wells, R. Cobordism groups of immersions. Topology 5 (1966), 281-294. 